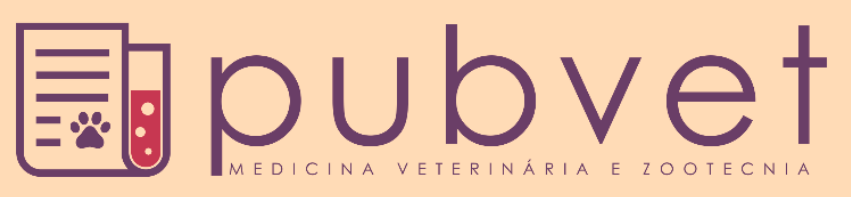

https://doi.org/10.31533/pubvet.v16n01a1007.1-5

\title{
Diagnóstico de disfunção da pars intermedia da pituitária em equino: Relato de caso
}

\author{
Luzia Leon Coelho Leal*\$, Kennya Mansiero Sanson Couto Souto Maior ${ }^{\circledast}$, Flávia Umpierre \\ Bueno ${ }^{\oplus}$, Carlos Afonso de Castro Beck $\%$
}

Universidade Federal do Rio Grande do Sur

*Autor para correspondência,E-mail: luzialcleal@hotmail.com

\begin{abstract}
Resumo. A Disfunção da Pars Intermedia da Pituitária (PPID), também chamada de doença de Cushing equina, é o distúrbio endócrino mais comum dos equídeos idosos. É observada uma combinação variável de sinais clínicos, sendo o mais comum o hirsutismo. $\mathrm{O}$ diagnóstico baseia-se nos sinais clínicos apresentados e em testes laboratoriais da concentração basal de ACTH ou o teste de supressão por dexametasona, sendo os mais comumente usados. Neste trabalho, relata-se o diagnóstico de PPID em uma égua, crioula, 24 anos, por meio do teste de supressão por dexametasona e hirsutismo como único sinal clínico.
\end{abstract}

Palavras-chave: Hirsutismo, dexametasona, doença neurodegenerativa, disfunção da pars intermédia da pituitária (PPID)

\section{Diagnosis of pituitary pars intermedia dysfunction in equine: case report}

\begin{abstract}
Pituitary Pars Intermedia Dysfunction (PPID), also called equine Cushing's disease, is the most common endocrine disorder of elderly horses. A variable combination of clinical signs is observed, the most common being hirsutism. The diagnosis is based on the clinical signs presented and laboratory tests of the baseline ACTH concentration or the dexamethasone suppression test, the most commonly used ones. In this study, the diagnosis of PPID in a mare, Creole, 24 years old, is reported by means of the dexamethasone suppression test and hirsutism as the only clinical sign.
\end{abstract}

Keywords: Hirsutism, dexamethasone, neurodegenerative disease, pituitary pars intermedia disfunction

\section{Introdução}

A Disfunção da Pars Intermedia da Pituitária (PPID) também conhecida como Síndrome de Cushing é uma endocrinopatia bastante comum na espécie equina, podendo acometer de 15 a $30 \%$ dos animais idosos, sem predileção por sexo (Del Pino et al., 2017; Hatazoe et al., 2015; Monteiro, 2015). A idade média dos animais afetados é de 18 a 23 anos, embora haja relato da doença em fêmeas com menos de sete anos (Franco et al., 2021; Hatazoe et al., 2015). Se trata de uma condição progressiva e lenta, devido a presença de adenoma, hiperplasia ou hipertrofia a nível da pars intermedia da pituitária (Del Pino, 2009; Franco et al., 2021; Smets et al., 2010).

Os sinais clínicos são pouco específicos e os mais relatados são hirsutismo, obesidade, laminite, infertilidade (Del Pino, 2009; Elliott, 2001; Kooistra \& Galac, 2012; Pereira et al., 2001), poliuria e polidpsia (Hatazoe et al., 2015), perda de peso, letargia, queda de performance (Arnaldi et al., 2003), hiperidrose, catabolismo muscular, redistribuição de peso (resultando na perda da musculatura epaxial e abdome pendular) e infecções secundárias e/ou cicatrização retardada também são comumente encontrados (McGowan, 2013). Os cavalos também podem apresentar uma variedade de condições 
secundárias que se desenvolvem como consequência da imunossupressão, incluindo doença periodontal, doença de linha branca e abscessos únicos (Dybdal et al., 1994).

Diagnosticar um caso de síndrome de cushing em equinos pode ser um desafio, embora um diagnóstico presuntivo possa ser feito com base nos sinais clínicos e dados laboratoriais. O teste inicial deve incluir hemograma completo, perfil bioquímico e urinálise. Testes adicionais podem colaborar na confirmação do diagnóstico, como por exemplo, teste de supressão por dexametasona (Elliott, 2001). A concentração basal de ACTH ou o teste de baixa dose de estimulação com dexametasona são mais comumente usados (McGowan, 2013).

\section{Relato de caso}

Foi atendido no hospital de clínicas veterinárias da Universidade Federal do Rio Grande do Sul um equino, fêmea, da raça crioula, com 24 anos de idade. A principal queixa do tutor era que a égua apresentava um crescimento de pelo anormal há aproximadamente três anos.

Suspeitando-se de um caso de Síndrome de Cushing a anamnese consistiu em indagações quanto a sinais de laminite, letargia, poliúria e polidpsia, mas o tutor não observou alterações. O animal não possuía histórico reprodutivo. Sua alimentação consistia em feno de alfafa três vezes ao dia, ração dois $\mathrm{kg}$ duas vezes ao dia e água a vontade. Vermifugação e vacinação em dia.

Ao exame clínico o animal apresentava FC 36 bpm, FR $12 \mathrm{mpm}$, mucosas róseas, tempo de preenchimento capilar de dois segundos, temperatura retal $38,4^{\circ} \mathrm{C}$. Escore corporal 5 (escala de 1 a 9), deposição de gordura na base da cauda e supra orbital, abdômen pendular e hirsutismo (Figura 1).

Foi realizado hemograma o qual apresentou hematócrito de 40\% (29 a 43\%), eritrócitos 7.8 (7.5 a 10) e hemoglobina de 14.7 (10 a 14); no leucograma observou-se leucócitos totais 7100 (5800 a 13200), com linfopenia de 1704 (2000 a 7500), proteína plasmática 72 g/L. No exame bioquímico apenas a CK estava aumentada; demais enzimas, proteínas e metabólitos estavam normais.

Para diagnóstico confirmatório o animal foi submetido ao teste de supressão por dexametasona, apresentando resultado basal de 3,5 MCG/dL; 15 horas após supressão 3,2 MCG/dL e após 19 horas de supressão 3,7 MCG/dL, como mostra a figura 2.

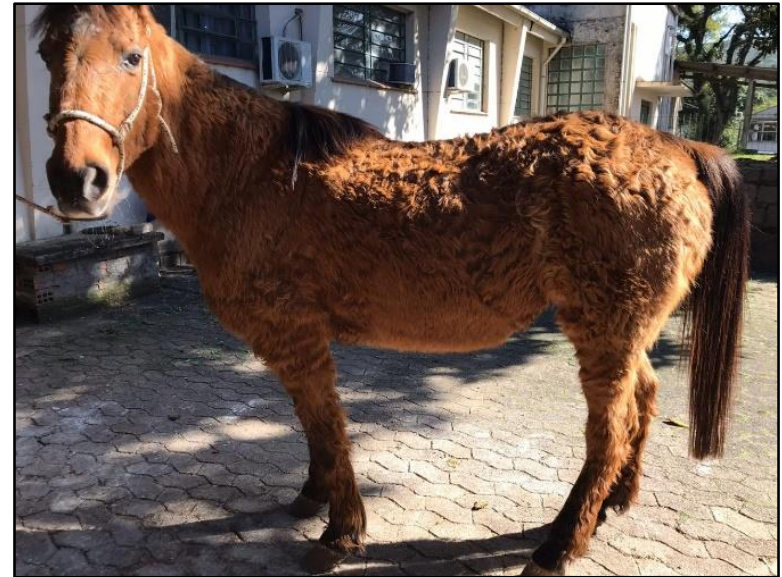

Figura 1. Composição corporal do animal.

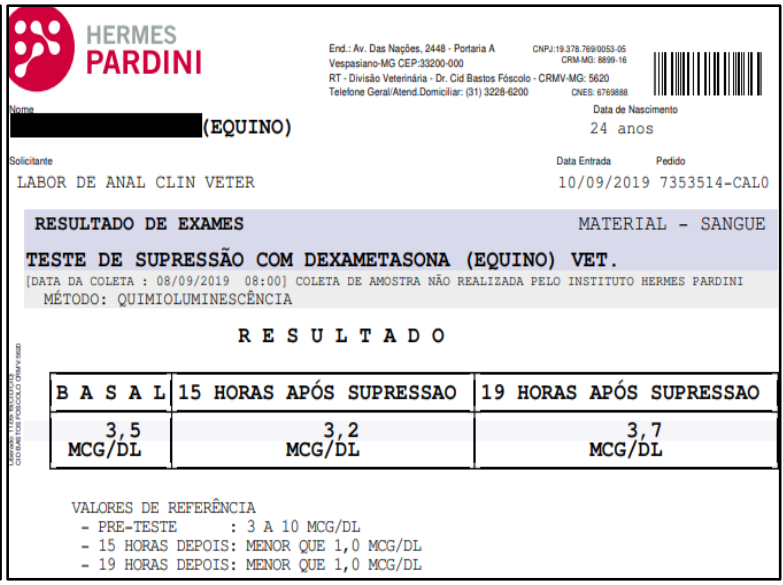

Figura 2. Resultados do exame laboratorial.

\section{Discussão}

A PPID é uma doença neurodegenerativa associada ao envelhecimento (McGowan, 2013). No relato foi acompanhado o caso de uma égua idosa com 24 anos de idade. Os cavalos mais velhos têm maior probabilidade de desenvolver PPID porque a inibição dopaminérgica diminui com a idade. Os neurônios dopaminérgicos se estendem dos núcleos periventriculares dentro do hipotálamo até a pars intermediária da glândula pituitária e produzem dopamina. A dopamina secretada por esses neurônios se liga aos receptores D2 nos melanotróficos e inibe sua atividade. A perda de neurônios dopaminérgicos por dano oxidativo é um processo normal de envelhecimento, mas a degeneração é acelerada em alguns cavalos, e esses animais são suscetíveis a PPID (Del Pino, 2009; Del Pino et al., 2017). 
O diagnóstico de PPID é baseado na história, sinais clínicos e achados laboratoriais, podendo ser mais comumente realizado pela observação de hirsutismo e outros sinais clínicos em equídeos mais velhos (Hatazoe et al., 2015; Schott, 2002). O hirsutismo não é uma característica normal do envelhecimento e é patognomônico para a PPID, ele por sua vez pode variar de uma queda tardia a cada ano, uma pelagem irregular com manchas de alopecia, até uma pelagem permanente longa ou até encaracolada (McGowan, 2013). Assim, como afirmam McGowan et al. (2012) o único sinal clínico observado nessa paciente foi o histórico de hirsutismo, destacando a importância desse sinal clínico.

Nos exames laboratoriais de hematologia e bioquímica não foram observadas alterações importantes que explicassem o diagnóstico de PPID. Segundo McGowan (2013), os exames de rotina citados são pouco úteis no diagnóstico de PPID, uma vez que os cavalos com PPID não apresentam diferença significativa em relação aos cavalos normais com a mesma idade. A alteração vista no leucograma de linfopenia é vista em cavalos idosos, independentemente de terem ou não PPID. Alterações inflamatórias, se presentes, representam doenças concomitantes e não os efeitos da hipercortisolemia (McGowan, 2013).

A presença de idade avançada e sinais clínicos podem ser considerados adequados para um diagnóstico clínico em campo. No entanto, os testes laboratoriais também são importantes por várias razões, incluindo a investigação de casos ambíguos, casos subclínicos, diferenciação da síndrome metabólica equina, bem como para monitorar a eficácia do tratamento (Durham et al., 2014). Uma variedade de testes laboratoriais de diagnóstico está disponível, mas uma limitação é que um teste padrão-ouro ante mortem ainda não foi identificado. Um diagnóstico definitivo pode ser alcançado pelo exame post-mortem em combinação com sinais clínicos, mas isso é de valor limitado para o clínico praticante (McGowan, 2013). O diagnóstico é melhor apoiado pela manifestação de sinais clínicos característicos e resultados de testes endocrinológicos, de preferência um resultado de teste de supressão com dexametasona de suporte (Schott, 2002).

Os testes de diagnóstico atuais para PPID que são considerados com precisão e disponibilidade adequadas para uso clínico incluem a concentração do hormônio adrenocorticotrópico plasmático basal (ACTH), o teste noturno de supressão da dexametasona (ODST) e o teste de estimulação do hormônio liberador de tireotropina (TRH) (medição do ACTH) (Durham et al., 2014). No presente trabalho foi utilizado para confirmação do diagnóstico o teste de supressão por dexametasona, proposto por Hatazoe et al. (2015), que consiste na medição de cortisol basal e após 15 e 19 horas da aplicação de dexametasona (40 $\mu \mathrm{g} / \mathrm{kg}$, IM). O objetivo do teste é detectar uma falha na supressão do cortisol após a administração de dexametasona em cavalos com PPID. A lógica é que a ACTH e a produção resultante de cortisol adrenal dos cavalos afetados não são afetadas pelo feedback negativo. A pars intermedia não é afetada pelo feedback negativo; portanto, os cavalos afetados não mostram uma supressão do cortisol após a administração do glicocorticoide exógeno, dexametasona (McGowan, 2013). Assim como visto no relato de Hatazoe et al. (2015), a égua atendida no HCV teve concentração do cortisol superior nas 15 e 19 horas após aplicação, o que confirmou o diagnóstico de PPID.

Existem vários fatores a serem considerados em relação a se e quando tratar. A terapia médica melhora a qualidade de vida dos cavalos afetados. É importante o cuidado preventivo geral e o aprimoramento da administração dos proprietários como adjuvante da terapia médica, incluindo cuidados com os cascos, desparasitação regular, grossamento e cuidados dentários. Atenção à dieta também é importante, cavalos com PPID estão envelhecidos e potencialmente em estado catabólico, por isso é importante fornecer uma dieta balanceada com proteínas de alta qualidade e suplementar os minerais e vitaminas (McGowan, 2013).

No passado, o tratamento médico de PPID baseava-se em três classes básicas de medicamentos: Inibidores do cortisol (trilostano), antagonistas da serotonina (cloridrato de ciproheptadina) e agonistas da dopamina (pergolida e bromocriptina). A pergolida é atualmente o medicamento mais usado, pois atua como um agonista do receptor de dopamina. Os relatos demonstram sua capacidade de reduzir significativamente a concentração plasmática de ACTH em cavalos com PPID. A pergolida deve ser administrada por via oral, na dose de $0,5 \mathrm{mg} /$ equino, uma vez por dia, durante quatro a seis semanas. A ciproheptadina também tem sido utilizada com sucesso no tratamento de cavalos com síndrome de cushing, sendo administrada, inicialmente, na dose de $0,25 \mathrm{mg} / \mathrm{kg}$ diariamente, por duas a três semanas, 
podendo ser aumentada para duas vezes ao dia durante o primeiro mês se não houver modificação dos sinais clínicos (Del Pino et al., 2017; Franco et al., 2021; Kastelan et al., 2009; Smets et al., 2010). No caso relatado o tratamento foi impossibilitado por dificuldade de acesso as drogas, além disso, não haviam alterações clínicas significativas na paciente que tornassem imprescindível o tratamento.

\section{Conclusão}

A Disfunção da Pars Intermedia da Pituitária (PPID) também conhecida como Síndrome de Cushing é uma doença neurodegenerativa associada ao envelhecimento em equinos. Os sinais clínicos são pouco específicos, sendo o mais comum o hirsutismo, também podem estar associadas a essa síndrome doenças concomitantes como laminite, infecções secundárias e infertilidade. Diante disso, se torna importante o uso de exames laboratoriais para a confirmação do diagnóstico, a concentração basal de ACTH ou o teste de baixa dose de estimulação com dexametasona são mais comumente usados. $\mathrm{O}$ tratamento da PPID no Brasil ainda é difícil e oneroso, pois a principal medicação utilizada não é encontrada no país, o que impede a recuperação dos animais acometidos.

\section{Referências bibliográficas}

Arnaldi, G., Angeli, A., Atkinson, A. B., Bertagna, X., Cavagnini, F., Chrousos, G. P., Fava, G. A., Findling, J. W., Gaillard, R. C., \& Grossman, A. B. (2003). Diagnosis and complications of Cushing's syndrome: a consensus statement. The Journal of Clinical Endocrinology \& Metabolism, 88(12), 5593-5602. https://doi.org/10.1210/jc.2003-030871.

Del Pino, F. J. G. (2009). Síndrome de Cushing en Equinos (Equine Cushing's. REDVET, 10(7).

Del Pino, T. S., Pazinato, F. M., \& Curcio, B. R. (2017). Síndrome de Cushing em égua da raça crioula - relato de caso.

Durham, A. E., McGowan, C. M., Fey, K., Tamzali, Y., \& Van der Kolk, J. H. (2014). Pituitary pars intermedia dysfunction: diagnosis and treatment. Equine Veterinary Education, 26(4), 216-223.

Dybdal, N. O., Hargreaves, K. M., Madigan, J. E., Gribble, D. H., Kennedy, P. C., \& Stabenfeldt, G. H. (1994). Diagnostic testing for pituitary pars intermedia dysfunction in horses. Journal of the American Veterinary Medical Association, 204(4), 627-632.

Elliott, M. (2001). Cushing's Disease: a new approach to therapy in equine and canine patients. British Homoeopathic Journal, 90(1), 33-36.

Franco, F. A., Santos, F. C. C., Costa, G. V., Oliveira, H. R., Ugolini, L. W., Bondan, C., \& Alves, L. P. (2021). Conduta diagnóstica em equino da raça Crioula com síndrome de Cushing. Acta Scientiae Veterinariae, 49(1), 598.

Hatazoe, T., Kawaguchi, H., Hobo, S., \& Misumi, K. (2015). Pituitary pars intermedia dysfunction (equine Cushing's disease) in a Thoroughbred stallion: a single report. Journal of Equine Science, 26(4), 125-128.

Kastelan, D., Dusek, T., Kraljevic, I., Polasek, O., Giljevic, Z., Solak, M., Salek, S. Z., Jelcic, J., Aganovic, I., \& Korsic, M. (2009). Hypercoagulability in Cushing's syndrome: the role of specific haemostatic and fibrinolytic markers. Endocrine, 36(1), 70-74. https://doi.org/10.1007/s12020-0099186-y.

Kooistra, H. S., \& Galac, S. (2012). Recent advances in the diagnosis of Cushing's syndrome in dogs. Topics in Companion Animal Medicine, 27(1), 21-24. https://doi.org/10.1053/j.tcam.2012.06.001.

McGowan, C. (2013). Hyperadrenocorticism (Pituitary Pars Intermedia Dysfunction) in horses. Clinical Endocrinology of Companion Animals, 11, 100-114.

McGowan, T. W., Pinchbeck, G. P., \& McGowan, C. M. (2012). Prevalence, risk factors and clinical signs predictive for equine pituitary pars intermedia dysfunction in aged horses. Equine Veterinary Journal, 45(1), 74-79.

Monteiro, R. M. S. F. L. (2015). Abordagem à disfunção da PARS Intermedia da glândula pituitária de equinos. Universidade Federal do Rio Grande do Sul. 
Pereira, M. A. A., Araújo, R. S., \& Bisi, H. (2001). Síndrome de Cushing associada à hiperplasia macronodular das adrenais: apresentação de um caso e revisão da literatura. Arquivos Brasileiros de Endocrinologia \& Metabologia, 45(6), 619-627.

Schott, H. C. (2002). Pituitary pars intermedia dysfunction: equine Cushing's disease. Veterinary Clinics: Equine Practice, 18(2), 237-270.

Smets, P., Meyer, E., Maddens, B., \& Daminet, S. (2010). Cushing's syndrome, glucocorticoids and the kidney. General and Comparative Endocrinology, 169(1), 1-10. https://doi.org/10.1016/j.ygcen.2010.07.004.

Histórico do artigo:

Recebido: 17 de setembro de 2021

Aprovado: 22 de outubro de 2021

Disponível online: 13 de dezembro de 2021
Licenciamento: Este artigo é publicado na modalidade Acesso Aberto sob a licença Creative Commons Atribuição 4.0 (CC-BY 4.0), a qual permite uso irrestrito, distribuição, reprodução em qualquer meio, desde que o autor e a fonte sejam devidamente creditados. 